\title{
Casein phosphopeptide-amorphous calcium phosphate products in caries prevention
}

\author{
Abstracted from \\ Raphael S, Blinkhorn A. \\ Is there a place for Tooth Mousse in the prevention and treatment of early dental caries? \\ A systematic review. BMC Oral Health 2015; 15: 113. DOI :10.1186/s12903-015-0095-6 \\ Address for correspondence: Department of Population Oral Health, Faculty of Dentistry, \\ The University of Sydney, 1 Mons Road, Westmead, NSW 2145, Australia. E-mail Sarah.raphael@sydney.edu.au
}

\section{Question: Is there sufficient clinical evidence available to support the use of Tooth Mousse ${ }^{\circledR}$ (MI Paste ${ }^{\circledR}$ ) and Tooth Mousse Plus ${ }^{\circledR}$ (MI Paste Plus ${ }^{\circledR}$ ) over a routine oral care regimen for the prevention and treatment of early dental caries?}

Data sources Medline, Embase, PreMedline and Cochrane Central Register of Controlled Trials.

Study selection Clinical trials, investigating only clinical caries outcomes on participants of any age comparing the use of Tooth Mousse ${ }^{\circledR}$ or Tooth Mousse Plus ${ }^{\circledR}$ to a routine oral care regimen for the prevention of dental caries with or without comparison to additional preventive products. Studies that used other formulations of CCP-ACP were excluded. Trials using split-mouth design were also excluded. Only English language papers were considered.

Data extraction and synthesis Papers were assessed independently by both authors using the Cochrane Collaboration tool for assessing risk of bias in randomised trials. ${ }^{1}$ Meta-analysis was not possible due to study heterogeneity.

Results Twelve studies were available for the final review; three studies on caries prevention were assessed as having low risk of bias. The remaining nine studies, with high risk of bias, reported on treatment or regression of early carious lesions. Two RCTs reported no significant benefits in the use of Tooth Mousse ${ }^{\circledR}$ over brushing with a fluoride toothpaste, a third study demonstrated a statistically significant increase in enamel microhardness for the Tooth Mousse ${ }^{\circledR}$ group compared to control group but no difference to the group who had fluoride gel treatment. Overall the authors concluded that Tooth Mousse ${ }^{\circledR}$ performs no better than fluoride in the prevention of carious lesions.

Seven of nine studies evaluated caries lesion severity in orthodontic patients, with four demonstrating statistically significant white spot regression. Two trials in non-orthodontic patients showed statistically significant remineralising potential of Tooth Mousse ${ }^{\circledR}$ over fluoride mouthrinse or the regular use of fluoride toothpaste in 14-30 days. Conclusions The authors found no evidence to support the use of Tooth Mousse ${ }^{\circledR}$ over brushing with a fluoride toothpaste for the prevention of early caries. ToothMousse ${ }^{\circledR}$ appeared to benefit regression of white spot lesions associated with orthodontic treatment but the evidence is limited. Effectiveness of Tooth Mousse ${ }^{\circledR}$ was not significantly increased by the addition of fluoride in Tooth Mousse Plus $®$ and evidence is still lacking to support the use of one over another. High quality randomised clinical trials are needed before these products can be recommended for the prevention and treatment of early carious lesions in the general population. Source of funding The publication was funded by Colgate Palmolive, Australia.

\section{Commentary}

Casein phosphopeptide-amorphous calcium phosphate (CPP-ACP) has a potential to promote remineralisation by stabilising high concentrations of calcium and phosphate ions at the tooth surface. ${ }^{2}$ This remineralising ability has been demonstrated in vitro ${ }^{3-5}$ and in situ ${ }^{6-8}$ studies and led to the development of Tooth Mousse ${ }^{\circledR}$ and Tooth Mousse Plus ${ }^{\circledR}$ (GC Corporation). However, clinical trials are limited. CPP-ACP products are contraindicated in children with milk allergy and the use of Tooth Mousse Plus® is not recommended in children younger than six years due to the fluoride content.

This well-conducted review sought to answer a clearly focused question related to the clinical effectiveness of two CPP-ACP products; Tooth Mousse ${ }^{\circledR}$ and Tooth Mousse Plus ${ }^{\circledR}$, limiting the evaluation only to those studies that strictly followed the manufacturer's instructions. Inclusion criteria were clearly defined for the evaluated studies in terms of participants, study design, intervention, comparison and outcome measures.

The search strategy however introduced a potential for language and publication bias. Several but not all scientific databases were searched; for example CINAHL, ISI web of knowledge, Conference Index papers and others. Only publications in English were included. Unfortunately grey literature was not searched and in the review, it seems that key people were not contacted, if necessary to identify missed, unpublished or in-progress papers. In addition the authors did not mention if the reference lists of the chosen articles were scanned to pick up additional papers that may be published globally. It is possible therefore that papers may have been omitted from the review.

Twelve clinical trials dating from 2009 to 2013 were included in the final review with only two being double-blind randomised controlled trials. Trials with crossover design were excluded due to the obvious limitation of cross contamination with the control sites. The authors also excluded trials that did not follow manufacturer's instructions, but they did not indicate how many studies fell into this category.

Three studies reported caries prevention and nine studies reported regression or treatment of early caries. The reviewers used the Cochrane Collaboration's tool for assessing risk of bias, however 
it may have also been beneficial for them to consider utilisation of the GRADE tool $^{9}$ to enable the reader to easily compare quality of the included studies. Study details and findings were provided in easy-to-read tables, however in this review it perhaps could have aided clarity to include a number of additional categories within the study review. An additional category for example that could have been included in the table was the location of each individual study, a factor that can significantly influence the comparability and external validity of the results.

Like many clinical reviews there was considerable heterogeneity in the type of studies included. The studies were highly variable in terms of participants age (0-31 years) and number, duration of study (from two weeks to six months) and the outcome measures (clinical indices, enamel microhardness, laser and light-induced fluorescence and visual scoring of photographs).The intervention and control groups also varied among studies. Three studies directly compared Tooth Mousse ${ }^{\circledR}$ to a control group and two studies used placebo crème. The remaining studies compared Tooth Mousse ${ }^{\circledR}$ or Tooth Mousse Plus ${ }^{\circledR}$ to other products with or without control, including fluoride toothpaste/mouthrinse/gel/varnish, chlorhexidine gel and microabrasion. The authors recognised that the diversity of studies precluded quantitative assessment of findings and also limited any definitive conclusion.

Within clinical trials, effects of confounding factors such as diet, saliva and fluoride cannot be avoided and these could have impacted on or altered the outcome. Furthermore, it would have been useful from a cost-effective perspective if the caries baseline level was recorded in the included trials as this would have helped provide better insight about caries experience among participants. Apparently, and as some studies showed, preventive measures are likely to be more cost-effective in the high caries risk population; for example there is evidence that fissure sealants are more cost effective in this group. ${ }^{10}$ Likewise, with regard to the caries preventive effects of fluoride toothpaste, the number needed to treat (NNT) varied with the level of disease in the population, being most favourable in communities with a high level of the disease. ${ }^{11}$

Tooth Mousse products are quite expensive compared to alternative preventive agents. Within the UK, a $35 \mathrm{ml}$ tube of GC Mousse currently costs approximately $£ 12$ for once- or twice-daily application. This compares with the cost of Duraphat fluoride varnish $(£ 27)$ for a $10 \mathrm{ml}$ tube for twice yearly application or a regular fluoride toothpaste averaging $£ 3$ for a $100 \mathrm{ml}$ tube. ${ }^{12}$ From our perspective, Tooth Mousse products would need to have a significantly strong evidence base demonstrating considerable effectiveness to warrant its cost.

Globally significant research is funded by commercial organisations and we know that such research may report biased results and should be read with caution. ${ }^{13}$ The Cochrane commercial sponsorship policy $(2014)^{14}$ states that, to minimise bias neither authors nor any part of the review should be funded by pharmaceutical companies or similar organisations. In this review the research is funded interestingly by Colgate, an organisation which does not produce a CPP-ACP product. Despite the fact that this review seems very comprehensive, the reader should be aware that a review which does not suggest a benefit for CPP-ACP can only improve the appeal of non CPP-ACP products, for example Colgate products.

No side or adverse effects of Tooth Mousse ${ }^{\circledR}$ and Tooth Mousse Plus ${ }^{\circledR}$ were reported in the included papers except for non serious gastrointestinal symptoms recorded for one participant in one study.

It is surprising that the newer product, Tooth Mousse Plus®, containing $900 \mathrm{ppm}$ fluoride did not significantly affect the clinical outcomes despite the synergistic effect demonstrated in in vitro and in situ studies. ${ }^{15-17}$

There is insufficient evidence to support the clinical effectiveness of Tooth Mousse ${ }^{\circledR}$ and Tooth Mousse Plus ${ }^{\circledR}$ over routine use of fluoride. The use of the traditional preventive measures that are supported by high quality clinical evidence, eg topical fluoride and fissure sealants, should continue until the evidence related to newer preventive activities is improved both in quality and quantity.

Thikrayat Bani Hani, Anne C. O'Connell and Brett Duane Dublin Dental University Hospital, Dublin, Eire

1. Higgins JPT, Altman DG and Sterne JAC (eds). Chapter 8. Assessing risk of bias in included studies. In: Cochrane Handbook for Systematic Reviews of Interventions Version 5.1.0. Edited by Higgins JPT and Green S. The Cochrane Collaboration; March 2011. http://handbook.cochrane.org/chapter_8/8_assessing_risk_of_bias_in_ included_studies.htm [Accessed 12 June 2016]

2. Reynolds EC. Calcium phosphate-based remineralization systems: scientific evidence? Aust Dent / 2008; 53: 268-273.

3. Oshiro M, Yamaguchi K, Takamizawa T, et al. Effect of CPP-ACP paste on tooth mineralization: an FE-SEM study. J Oral Sci 2007; 49: 115-120.

4. Yamaguchi K, Miyazaki M, Takamizawa T, Inage H, Kurokawa H. Ultrasonic determination of the effect of casein phosphopeptide-amorphous calcium phosphate paste on the demineralization of bovine dentin. Caries Res 2007; 41: 204-207.

5. Poggio C, Lombardini M, Vigorelli P, Ceci M. Analysis of dentin/enamel remineralization by a CPP-ACP paste: AFM and SEM study. Scanning 2013; 35: 366-374.

6. Cai F, Shen P, Morgan MV, Reynolds EC. Remineralization of enamel subsurface lesions in situ by sugar-free lozenges containing casein phosphopeptide-amorphous calcium phosphate. Aust Dent / 2003; 48: 240-243.

7. Cai F, Shen P, Morgan MV, Reynolds EC. Remineralization of enamel subsurface lesions in situ by sugar-free lozenges containing casein phosphopeptide-amorphous calcium phosphate. Aust Dent / 2003; 48: 240-243.

8. Manton DJ, Walker GD, Cai F, Cochrane NJ, Shen P, Reynolds EC. Remineralization of enamel subsurface lesions in situ by the use of three commercially available sugar-free gums. Int J Paediatr Dent 2008; 18: 284-290.

9. http://www.gradeworkinggroup.org/ [Accessed 12 June 2016]

10. Neusser S, Krauth C, Hussein R, Bitzer EM. Clinical effectiveness and cost-effectiveness of fissure sealants in children and adolescents with a high caries risk. GMS HealthTechnol Assess 2014; 10: Doc02. doi: 10.3205/hta000118. eCollection 2014.

11. Marinho VC, Higgins IP, Sheiham A, Logan S. Fluoride toothpastes for preventing dental caries in children and adolescents. Cochrane Database Syst Rev 2003; (1): CD002278.

12. https://www.henryschein.co.uk/ [Accessed 12 June 2016]

13. Jørgensen AW, Hilden J, Gøtzsche PC. Cochrane reviews compared with industry supported meta-analyses and other meta-analyses of the same drugs: systematic review. Br Med J 2006; 333: 782.

14. http://community-archive.cochrane.org/editorial-and-publishing-policy-resource/ conflicts-interest-and-cochrane-reviews [Accessed 12 June 2016]

15. ElSayad I, Sakr A, Badr Y. Combining casein phosphopeptide-amorphous calcium phosphate with fluoride: synergistic remineralization potential of artificially demineralized enamel or not? J Biomed Opt 2009; 14: 044039. doi: $10.1117 / 1.3210780$.

16. Srinivasan N, Kavitha M, Loganathan SC. Comparison of the remineralization potential of CPP-ACP and CPP-ACP with 900ppm fluoride on eroded human enamel: An in situ study. Arch Oral Biol 2010; 55: 541-544.

17. Wu G, Liu X, Hou Y. Analysis of the effect of CPP-ACP tooth mousse on enamel remineralization by circularly polarized images. Angle Orthod. 2010; 80: 933-938

Evidence-Based Dentistry (2016) 17, 46-47. doi:10.1038/sj.ebd.6401168 